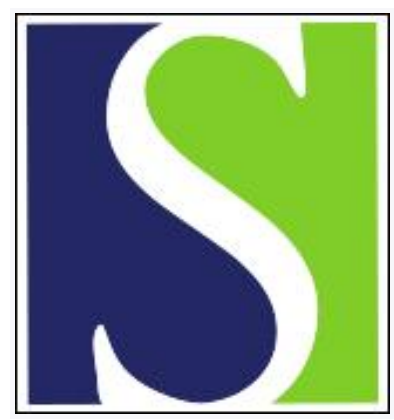

Scand J Work Environ Health 2007;33(3):223-232

https://doi.org/10.5271/sjweh.1131

Issue date: 30 Jun 2007

\title{
Cognitive function in outpatients with perceived chronic
} stress

by Öhman L, Nordin S, Bergdahl J, Slunga Birgander L, Stigsdotter Neely A

Affiliation: Department of Psychology, Umeå University, SE-901 87 Umeå, Sweden. lena.ohman@psy.umu.se]

The following articles refer to this text: 2012;38(4):358-369;

2014;40(6):569-581; 2022;48(1):71-80

Key terms: burnout; cognitive function; executive functioning; memory; outpatient; perceived chronic stress; self-assessment; self-report; work stress

This article in PubMed: www.ncbi.nlm.nih.gov/pubmed/17572832

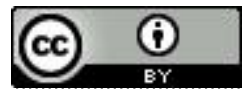




\title{
Cognitive function in outpatients with perceived chronic stress
}

\author{
by Lena Öhman, BA, ${ }^{1}$ Steven Nordin, PhD, ${ }^{1,2}$ Jan Bergdahl, PhD, ${ }^{1,3}$ Lisbeth Slunga Birgander, MD, ${ }^{4}$ Anna \\ Stigsdotter Neely, $P h D^{1}$
}

\begin{abstract}
Öhman L, Nordin S, Bergdahl J, Slunga Birgander L, Stigsdotter Neely A. Cognitive function in outpatients with perceived chronic stress. Scand J Work Environ Health 2007;33(3):223-232.

Objectives The number of employees on sick leave due to stress-related syndromes has increased drastically in many industrialized countries. Cognitive dysfunction is a common complaint among patients suffering from chronic stress. Previous research on chronic stress has mainly focused on its interpersonal, attitudinal, psychological, organizational, and physical effects. Less attention has been allocated to effects of chronic stress on cognitive functioning. Hence the aim of the present study was to investigate the relationship between chronic stress and cognition.

Methods The cognitive performance of 19 chronic stress outpatients and 19 matched controls was studied. A battery of standard and experimental cognitive tests assessing processing speed, attention, working memory, and episodic and semantic memory were administered.

Results Performance deficits of the chronic stress patients relative to the performance of the controls were found for some but not all cognitive tasks, indicating a selective deficit. Deficits were found for episodic memory, particularly for learning across repeated trials and for tasks requiring divided attention during either the encoding or retrieval of words. Performance differences were also found for aspects of working memory, mental tempo, semantic access, and prospective memory, but not for basic perceptual speed measures.

Conclusions These findings may suggest suboptimal executive functioning (eg, strategic or attentional control) among chronic stress patients. Particularly, poor performance in letter fluency and prospective memory were significantly associated with chronic stress, the finding suggesting the usefulness of these tests as clinical tools in evaluations of chronic stress states.
\end{abstract}

Key terms burnout; executive functioning; memory; work stress.

The number of employees on long-term sick leave due to prolonged exposure to high levels of work stress has increased drastically within the past few years in many industrialized countries (1). Chronic stress syndromes such as burnout is manifested in the form of such symptoms as emotional exhaustion, physical fatigue, tension, listlessness, and cognitive weariness (2) and sleeping disturbances (3) and can result in glucocorticoid dysregulation (4). Depression and anxiety are psychological problems that are integral parts of chronic stress, although the causal relation is unclear $(5,6)$. However, psychological conceptualization of work-related chronic stress overlaps with other medically unexplained disorders, for example, chronic fatigue syndrome (7), fibromyalgia (8), and multiple chemical sensitivity (9).
The similarities between the syndromes have been suggested to outweigh the differences and are apparent in case definitions and reported symptoms (10).

Despite the fact that chronic-stress-related syndromes have become a major concern for the authorities, there is still limited documentation of this condition in terms of symptoms, underlying mechanisms, agreed diagnostic criteria, and treatment (11). A common concern with respect to patients suffering from chronic stress syndromes such as burnout is that of reported cognitive problems, predominantly regarding memory and attention (12). Since intact cognitive functioning is of critical importance in our everyday lives, even mild deficits can be of great concern for the afflicted person, as well as for their families.

1 Department of Psychology, Umeå University, Umeå, Sweden.

2 Department of Occupational and Environmental Medicine, Örebro University Hospital, Örebro, Sweden.

3 Institute of Clinical Dentistry Faculty of Medicine, University of Tromsö, Tromsö, Norway.

4 Department of Public Health and Clinical Medicine, Umeå University, Umeå, Sweden.

Reprint request to: Dr A Stigsdotter Neely, Department of Psychology, Umeå University, SE-901 87 Umeå, Sweden. [E-mail: anna.neely@psy.umu.se] 
It has long been known that prolonged periods of physical and psychological stress have adverse effects on the structure and circuitry of the brain, notably the hippocampus and prefrontal cortex (13-15). Research examining the effects of chronic exposure of stress hormones on cognition has found impairment of declarative memory and aspects of working memory $(14,16)$, which are cognitive functions subserved by the medial temporal lobe, as well as by the prefrontal cortex.

Previous research on work-related chronic stress has mainly focused on its interpersonal, attitudinal, psychological, organizational, and physical effects (2). Less attention has been allocated to the effects of chronic stress on cognitive functioning. This state of affairs is unfortunate given the literature on the averse effects of stress on cognition $(16,17)$ and the reports of cognitive complaints among chronic stress patients. It is important to deepen our understanding about the consequences of work-related chronic stress on behavior in general and on cognitive functions in particular, in that it may inform us about new approaches to the diagnosis and support rehabilitation of the persons in question. To our knowledge only two recent studies have investigated the cognitive functions of burnout patients $(12,18)$. Both of these studies showed that burnout was associated with deficits in select aspects of cognitive functioning, more specifically, in nonverbal episodic memory, mental speed, response inhibition, and sustained attention. Moreover, the conclusion drawn in these studies was that the pattern of cognitive deficits may be indicative of an executive control deficit. Executive control is commonly referred to as a set of cognitive processes underlying the regulation and control of thought and behavior $(19,20)$, and it has been frequently ascribed to the function of the prefrontal cortex (21). Compromised executive control may manifest itself in several ways, such as deficits in working memory, inhibition, divided attention, and strategic processing, and, accordingly, can lead to different expressions in a person's behavior, such as loss of thought, problem-solving difficulties, and forgetfulness. Noteworthy is that findings of deficient executive control have been shown in other stress-related patient populations, such as in those with posttraumatic stress disorder and chronic fatigue syndrome $(22,23)$.

The objective of our study was to add further understanding to the sparse literature on the chronic stresscognition relationship by comparing patients on sick leave due to work-related chronic stress with a control group. We decided to investigate this issue in two ways. First, we adopted a rather exploratory approach by administering a battery of standard neuropsychological tests selected to assess declarative memory (episodic and semantic memory), working memory, and attention-processing speed and a questionnaire on everyday memory failures. On the basis of previous findings $(12,18)$, we hypothesized that the stress group would be impaired on some, but not all, of these tests, the most marked impairment occurring in more executive demanding tasks. Many standard neuropsychological tests have been criticized for being insensitive in detecting very mild cognitive deficits in some patient populations, despite reported everyday memory problems in these patient groups (24). Therefore, we complemented this clinical approach by adding two experimentally derived tasks to investigate the possibility that executive control deficits may form a core feature in persons suffering from workrelated chronic stress, as suggested by Sandström et al (12) and Van der Linden et al (18). These tasks address episodic memory, which many chronically stressed persons express concern about. In the first test, a dual-task paradigm was used for which we examined the impact of divided attention on episodic memory performance. To perform two tasks at the same time requires the ability to exert control over the allocation of attention between the two tasks, and this need constitutes a problem when one's executive control processes are impaired. Several studies have shown that divided attention limits the ability to process information in a deep and elaborate fashion; in other words, it hampers strategic organization (25-27). Thus we expected that divided attention would have negative effects on episodic memory performance in both groups, but that the chronic stress patients would be disproportionately affected under divided attention conditions when compared with the controls. This situation would be in line with the presented argument concerning suboptimal executive control processes in chronic stress patients.

In the second task, on the recall of concrete nouns with selective reminding, we were interested in the ability to learn across trials. We argued that gain across trials benefits from the strategic organization (eg, deep and elaborate processing) of the to-be remembered information, and such organization calls for efficient executive control, particularly under a selective reminding procedure (28). [See the Methods section for details.] Hence we hypothesized that the chronic stress patients would show less gain across a trial than the controls would. Finally, we were interested in determining which cognitive measures are significantly associated with chronic stress.

\section{Study population and methods}

\section{Study population}

Altogether 19 outpatients, 13 women and 6 men, with perceived chronic stress were recruited from the Stress Clinic, Department of Occupational and Environmental Medicine, Umeå University, Sweden (table 1). The patient population at the Stress Clinic had initially been 
diagnosed with chronic stress by physicians in primary care and referred to the Stress Clinic for evaluation for participation in a 1-year cognitive-behavioral stress management group program. The Shirom Melamed Burnout Questionnaire (SMBQ) (29) was used by the primary care physicians to evaluate perceived chronic stress, and the criterion for referral to the Stress Clinic was set at a SMBQ index of $>4.6$. The chronic stress was work-related for all of the patients, but a combination of stressors at work and outside work was common.

All of the participants were individually screened at the Stress Clinic in a structured interview for the following conditions, which also served as exclusion criteria: (i) other diseases that could cause fatigue or stress-related symptoms, cause future sick leave, or interfere with active participation, (ii) posttraumatic stress disorder, (iii) known drug abuse, (iv) need for individual therapy, (v) $>2$ years of unemployment, (vi) lack of fluency in Swedish, (vii) participation in any other ongoing study, and (viii) an age of $<25$ or $>55$ years. This screening was done by a treating physician and a clinical psychologist. Altogether 37\% did not fulfill the inclusion criteria, and $10 \%$ declined further participation.

From this pool of eligible patients, we invited 20 to participate in our study, of which one declined participation, 6 were medicated with selective-serotoninreuptake-inhibitor (SSRI) drugs, and 1 used hypnotic drugs for insomnia. All of the patients had an ongoing sick-leave period of 3-24 (mean 12.00) months.

Chronic stress was assessed according to the criteria set by Melamed et al (29) by using the SMBQ (29). The SMBQ is a validated questionnaire $(30,31)$ that consists of an overall index comprised of the following four subscales: burnout, tension, listlessness, and cognitive weariness. The internal consistencies (Cronbach's alpha) were high for the overall SMBQ index $(\alpha$ 0.98) and the SMBQ subscales $(\alpha$ 0.96, $\alpha$ 0.92, $\alpha$ 0.92, $\alpha$ 0.98 , respectively). The subscales were rated on a 7 point Likert scale, with the end points $1=$ almost never and $7=$ almost always. In a study performed by Grossi et al (30), a score of $\geq 3.75$ was classified as high stress and that of $\leq 2.75$ as low stress. All of the patients met the criteria for high stress according to these guidelines (mean 5.53 for the women and 5.72 for the men) when compared with the controls (mean 2.51 for the women and 1.92 for the men) (table 1).

A healthy control group, including 13 women and 6 men, was recruited from various occupational settings in Umeå, Sweden, and was matched to the patient group for gender, age, years of education and verbal ability (table 1). A Swedish, four-alternative multiple-choice synonym test was used to assess verbal ability (SRB: 1) (32). Separate analyses of variance (ANOVA) on each of these background variables indicated no group differences $(\mathrm{P}>0.10)$.
Table 1. Means and standard deviations for the background variables as a function of group.

\begin{tabular}{lcccc}
\hline Variable & \multicolumn{2}{c}{ Chronic stress group } & \multicolumn{2}{c}{ Control group } \\
\hline & Mean & SD & Mean & SD \\
\cline { 2 - 5 } & 39.16 & 6.37 & 39.89 & 6.72 \\
Age (years) & 13.47 & 2.33 & 13.37 & 2.13 \\
Education (years) & 22.58 & 3.93 & 24.42 & 2.61 \\
Verbal ability ${ }^{\text {a }}$ & $4.89^{c}$ & 0.85 & 2.04 & 0.59 \\
Shirom Melamed Burnout Questionnaire & & & \\
Burnout & $5.64^{\mathrm{c}}$ & 0.81 & 2.26 & 0.86 \\
Tension & $5.74^{\mathrm{c}}$ & 0.84 & 2.91 & 0.87 \\
Listlessness $_{\text {Cognitive weariness }}$ & $5.61^{\mathrm{c}}$ & 1.04 & 2.04 & 1.03 \\
Global & $5.59^{\mathrm{c}}$ & 0.73 & 2.33 & 0.70 \\
\hline
\end{tabular}

${ }^{a}$ A Swedish synonym test (SRB1; maximum $=30$ ).

b Subscales and global score.

c $\mathrm{P}<0.001$.

The study was conducted in accordance with the Declaration of Helsinki, and it was approved by the Ethics Committee of the Faculty of Medicine and Odontology, Umeå University (Umdnr 02-311). All of the participants gave their informed consent prior to their inclusion in the study.

\section{Subjective memory assessment}

Subjective memory concerns were assessed with the use of a 5-item questionnaire of everyday memory problems $(33,34)$. Each question was analyzed separately when the responses on each question served as the dependent measure. Questions 1, 4, and 5 were rated on a 5-point scale for which 1 indicated never and 5 represented very often. Items 2 and 3 were rated on a 5-point scale for which 1 indicated much better and 5 represented much worse. In table 2 the medians and ranges are presented for each of the five questions. In this study, the internal consistency (Cronbach's alpha) was high for the everyday memory problem questionnaire $(\alpha 0.90)$.

\section{Cognitive assessment}

All of the patients participated individually in an approximately 90 -minute test session. All of the cognitive tests were paper-and-pencil type. A 15-minute break was taken after half of the session. The order of cognitive task presentation was invariant for all of the participants, and the testing was done prior to the participants' inclusion in therapy.

Attention and processing speed. Six tasks were used to assess aspects of attention and mental speed. Digit symbol from WAIS-R (35) was used and administered according to standard procedures. The participants were required to transcribe as many symbols as possible 
Table 2. Medians and ranges for the self-reported memory problems as a function of group. (For questions 1,4 , and $5,1=$ never and 5 = very often; for questions 2 and 3, 1 = much better and 5 = much worse)

\begin{tabular}{|c|c|c|c|c|c|}
\hline & \multicolumn{2}{|c|}{ Chronic stress group } & \multicolumn{2}{|c|}{ Control group } & \multirow[t]{2}{*}{ U-value a } \\
\hline & Median & Range & Median & Range & \\
\hline 1. Does anyone close to you (family, friends) think you have a poor memory? & 4 & $2-5$ & 2 & $1-3$ & $29.00^{b}$ \\
\hline 2. How is your memory today in comparison with your memory 5 years ago? & 5 & $3-5$ & 3 & $1-5$ & $32.50^{b}$ \\
\hline $\begin{array}{l}\text { 3. How do you perceive your memory in comparison with that of other persons } \\
\text { your age? }\end{array}$ & 4 & $2-5$ & 3 & $1-4$ & $22.50^{\circ}$ \\
\hline $\begin{array}{l}\text { 4. Do you forget appointments if not prompted by someone else or by a reminder } \\
\text { such as a calendar or diary? }\end{array}$ & 4 & $1-5$ & 3 & $1-5$ & $64.50^{\mathrm{b}}$ \\
\hline 5. Does it occur that you do not remember things that have recently happened? & 4 & $2-5$ & 2 & $1-4$ & $22.00^{b}$ \\
\hline
\end{tabular}

a Mann-Whitney U test.

${ }^{b} \mathrm{P}<0.001$.

c $P<0.01$.

into empty boxes according to associations specified in a coding key. The key consisted of nine geometric symbols uniquely paired with digits from 1 to 9 . The number of items completed in 90 seconds was the indicator score.

Trail making test A (TMT A) was administered according to standard procedures $(36,37)$. The participants were asked to connect the numbers 1 to 25 consecutively as fast as possible. The time (seconds) to complete the test was used as the outcome measure.

Pattern comparison and letter comparison tasks (38) required the participants to make simple perceptual comparisons as rapidly as possible within a limited time period. For the pattern comparison task, the participants compared 30 pairs of line drawings consisting of three, six, or nine line segments and responded with the same or different judgments for each pair. The number of correct decisions for two separate 30-second trials was used as the indicator measure. Similarly, the letter comparison task required the participants to indicate whether pairs of letter strings (consisting of three, six, or nine letters) were the same or different. As before, the correct decisions for the separate 30 -second trials were used as the indicator measure.

Motor speed was determined by the number-copying and line-marking tests (39). Two forms of the numbercopying test contained 10 rows of 10 box pairs. Emphasizing speed, the participants were required to copy the number from the top box into the empty box below. The indicator measure was the mean number correct for both 30 -second tests. The line-marking test also consisted of 10 rows of 10 incomplete squares that were missing either a horizontal or vertical line. The participants were asked to work as quickly as possible, adding either a horizontal or vertical line to complete the square. The mean number of correct responses in 30 seconds was used as the indicator measure.

Working memory. Working memory was defined by four measures. Computational span (38) required storage and simultaneous processing during which the participants solved arithmetic problems while holding the final digit from each problem in their memory for later recall. The number of items increased from 1 to 7 , with three trials at each series length. The highest span (1 to 7) correctly recalled for two out of three trials was the outcome measure.

Two subtests of the WAIS-R (35), digit span forward and backward, were used and administered according to standard procedures. Forward and backward digit span were analyzed separately. As the outcome measure, the number of trials correctly completed was used.

Trail making test B (TMT B) was also administered according to standard procedures $(36,37)$. This task required storage, simultaneous processing, and the ability to shift. The participants were asked to alternate as quickly as possible between numbers and letters following the order of numbers and the alphabet. The time needed to complete (seconds) the text was used as the outcome measure.

Episodic memory. To measure the ability to explicitly recollect a previous episode, a free-recall task of 18 concrete nouns with selective reminding (40) was used. Each noun was presented orally at a rate of 5 seconds per noun. The participants were asked to memorize as many words as possible, with a free-recall test after the presentation of the last noun. In accordance with the selective reminding procedure, all of the to-be-remembered items were presented on the first trial $\left(\mathrm{T}_{1}\right)$. On each subsequent learning trial $\left(\mathrm{T}_{2}, \mathrm{~T}_{3}\right)$, only the items that were not recalled in the previous trial were presented again, and the participants were asked to recall all of the items. The order of presentation was determined by the original serial position in the list. A total of three trials was used. As the outcome measure, the number of items recalled in each trial was used.

In order to examine the effect of divided attention on episodic memory performance, we used four different lists, each consisting of 12 concrete nouns. 
A card-sorting task was used for distraction $(25,41)$; in this task the participants were required to sort standard playing cards into two piles according to their color (red versus black). The four test conditions were full attention at both encoding and retrieval (full-full), full attention at encoding and divided attention at retrieval (full-divided), divided attention at encoding and full attention at retrieval (divided-full), and divided attention at both encoding and retrieval (divided-divided). The order of the test conditions was counterbalanced. The participants were instructed to memorize the nouns that were orally presented at a rate of 2 seconds per noun (time was indicated by a beeper) followed by free recall (45 seconds). During the divided attention conditions, the participants were asked to sort one card each time a word was read (encoding phase), and at retrieval they were to sort one card at the sound of a beep. The outcome measure was the number of nouns recalled.

The Rey Complex Figure Test (RCFT) (42) was used to assess visual nonverbal memory. It was administered according to standard procedures. First, the participant was requested to copy a complex figure as correctly and quickly as possible. Thereafter, the participant was asked to redraw the figure twice from memory, first immediately after the copy trial and then again after a 30-minute delay (43), during which other tests were performed. The copy time and the number of correct recalled items, both immediately and at delayed recall, were used as the outcome measures.

The reminding task (44) taped prospective memory, the ability to remember to execute an intended action in the near future. The participants were told at the beginning of the test session to remind the experimenter to ask for their addresses at the end of the test session. The outcome measure was based on the amount of cue support needed to remember the action, (i) without a cue within 15 seconds, (ii) after a cue "Was there not something else we were supposed to do?", (iii) the person being asked what he or she was supposed to remember, and (iv) the person being retold what he or she was supposed to remember.

Semantic memory. A Swedish version of the Controlled Oral Word Association Test (COWAT) (45) was used to assess letter and category verbal fluency. In letter fluency, the participants were required to generate as many words as possible during 1 minute, beginning with the letters F, A, and S, with the exception of proper names, numbers, and words with the same suffix. In the category fluency task, the participants generated as many grocery food items, animals beginning with the letter $\mathrm{S}$, and occupations beginning with $\mathrm{B}$ during 1 minute. Both letter and category fluency was assessed after 30 seconds (early retrieval) and 60 seconds (late retrieval), and as the total score. Letter and category fluency was averaged across letters and categories, respectively, to form a composite score.

The presented tests were administered in the following order: (i) reminding task, instruction; (ii) RCFT, copy and immediate recall; (iii) digit symbol; (iv) recall of concrete words with selective reminding; (v) TMT A and B; (vi) digit span forward and backward; (vii) verbal ability, SRB 1; (viii) RCFT, delayed recall, followed by a 15-minute pause; (ix) computational span; (x) word recall and divided attention; (xi) pattern comparison, letter comparison; (xii) line marking, number copying; (xiii) letter and category verbal fluency; and (xiv) reminding task, recall.

\section{Results}

The results are presented in two main sections corresponding to the principal research questions. First, we determined whether the participants with chronic stress syndrome experienced more subjective memory problems than the controls. Second, we concentrated on whether the stress group performed at lower levels than the controls in cognitive tasks using a multivariate analysis of variance (MANOVA) followed by a univariate analysis of variance (ANOVA). Third, we used logistic regression to determine which of the 24 cognitive tests were significantly related to chronic stress.

\section{Subjective memory and cognitive performance}

First, as can be seen in table 2, the chronic stress patients experienced significantly more memory concerns on all five questions than the controls. This finding was confirmed by separate Mann-Whitney tests for each question $(\mathrm{P}<0.01)$.

Second, a 2 (group) $\times 24$ (cognitive tests) MANOVA, revealed a significant overall multivariate effect of group [Wilks' $\lambda=0.14, \mathrm{~F}(24,13)=3.46, \mathrm{P}<0.012, \eta^{2}=0.86$ ] indicating lower performance on several cognitive tasks for the chronic stress group. These significant findings are presented in the results under the respective cognitive domain and complemented with follow-up univariate ANOVA.

For illustrative purposes, the relative performance of the chronic stress patients across a task is displayed in figure 1 , portraying the means for the chronic stress group expressed in standard Z-scores. As can be seen from the figure, a pattern of preserved and inferior performance is present.

Attention and processing speed. The univariate ANOVA revealed no effects of group for the following tests: line marking $(\mathrm{P}<0.30)$, number copying $(\mathrm{P}<0.47)$, pattern comparison $(\mathrm{P}<0.07)$, letter comparison $(\mathrm{P}<0.12)$, or TMT A $(\mathrm{P}<0.20)$. However, digit symbol did not 


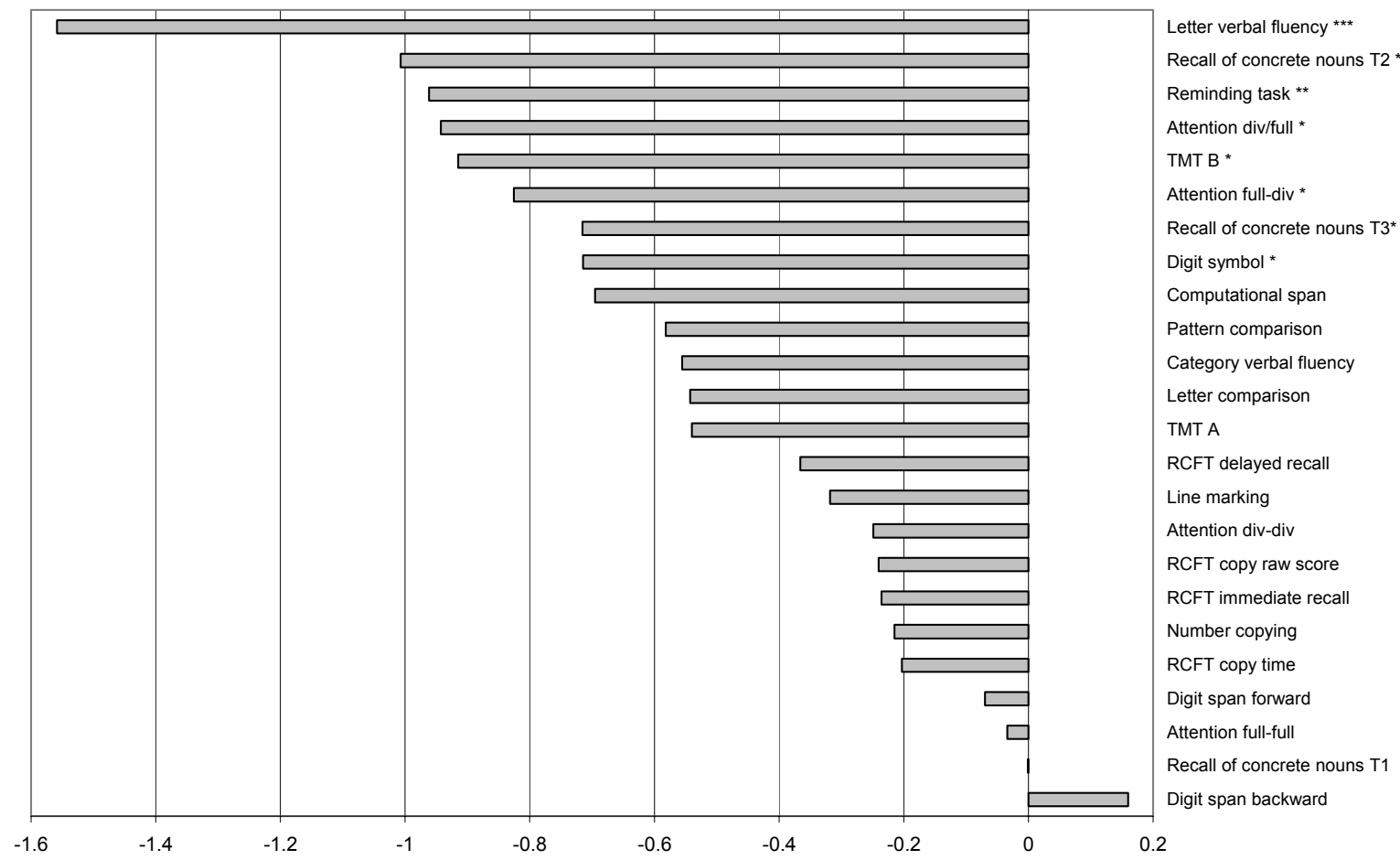

Note. ${ }^{*} \mathrm{P}<0.05, * * \mathrm{P}<0.01, * * * \mathrm{P}<0.001$

Figure 1. Z-scores for the chronic stress group relative to the control group across the cognitive tasks. $(T=$ trial, $T M T=$ trail making test, $\mathrm{RCFT}=$ Rey Complex Figure Test, div $=$ divided)

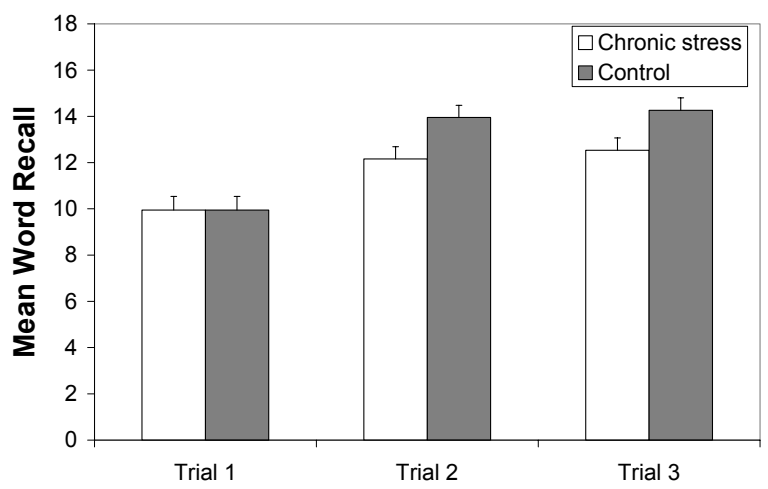

Figure 2. Means and standard errors of the means for recalled words with selective reminding as a function of group and trial.

conform to this pattern of results by showing that the performance of the chronic stress patients (mean 54.47) was significantly inferior $[\mathrm{F}(1,36)=5.15$, mean square error (MSE) 122.75, $\mathrm{P}<0.03$ ] to that of the controls (mean 62.63).

Working memory. The univariate analysis revealed a significant effect for TMT B $[\mathrm{F}(1,36)=4.53$, MSE 617.12, $\mathrm{P}<0.04$ ], reflecting that the chronic stress patients (mean 77.42) performed worse than the controls (mean 60.26). Their computation span was also close to significant $[\mathrm{F}$ $(1,36)=3.60$, MSE 11.10, $\mathrm{P}=0.06]$, showing that the performance of the chronic stress patients (mean 8.79) was inferior to that of the controls (mean 10.84). There were no group effects for digit span forward $(\mathrm{P}<0.84)$ or backward $(\mathrm{P}<0.64)$.

Episodic memory. The results from the selective reminding task for the recall of concrete nouns was further analyzed with a 2 (group: chronic stress, control) $\times 3$ (trial: $\mathrm{T}_{1}, \mathrm{~T}_{2}, \mathrm{~T}_{3}$ ) ANOVA with the repeated measure for the last factor. The means and standard errors of the means are shown in figure 2 . A significant main effect of trial $[\mathrm{F}(2,72)=61.63$, MSE 2.22, $\mathrm{P}<0.001]$ showed that performance improved significantly from $\mathrm{T}_{1}$ to $\mathrm{T}_{2}(\mathrm{P}<0.05)$, but not from $\mathrm{T}_{2}$ to $\mathrm{T}_{3}(\mathrm{P}>0.05)$. More interestingly, the interaction effect was significant $[\mathrm{F}(2,72)=4.42$, MSE $2.22, \mathrm{P}<0.01]$. The follow-up t-tests indicated that the control group gained significantly more from $\mathrm{T}_{1}$ to $\mathrm{T}_{2}$ than did the chronic stress group $(\mathrm{P}<0.05)$, whereas the gain from $T_{2}$ to $T_{3}$ was more or less identical for both groups $(\mathrm{P}>0.05)$. This pattern of results indicated that learning across trials was compromised among the chronic stress patients.

In studying whether the chronic stress patients, when compared with their controls, were differentially affected by divided attention at encoding, retrieval, or at both encoding and retrieval, we analyzed the performance data on the four episodic memory lists with a 2 (group: chronic stress, control) $\times 4$ (type of test: full-full, full-divided, divided-full, divided-divided) 
ANOVA with the repeated measure for the last factor. The means and standard errors are shown in figure 3. The main effect of the type of test was significant $[\mathrm{F}(3$, 108) $=19.69$, MSE 1.31, P<0.001] and indicated that the episodic memory performance decreased as a function of divided attention in such a way that the performance was best under the full-full (mean 6.34) condition, followed by the full-divided (mean 5.66), divided-divided (mean 4.68), and divided-full (mean 4.63) conditions, for which the two last conditions did not significantly differ from each other. Moreover, the quadratic group $x$ type of test interaction was significant $[\mathrm{F}(1,36)=4.84$, MSE 1.39, $\mathrm{P}<0.05]$, for which the follow-up t-tests indicated that the performance of both groups was equivalent in the full-full and divided-divided conditions $(\mathrm{P}>0.05)$, whereas the performance of the chronic stress group decreased more than that of the controls from the full-full condition to the divided-full condition $(\mathrm{P}<0.05)$, and marginally so from the full-full to the full-divided condition $(\mathrm{P}<0.10)$.

No group effects were observed for any of the RCFT tests: copy time $(\mathrm{P}<0.62)$, copy raw score $(\mathrm{P}<0.50)$, immediate recall $(\mathrm{P}<0.47)$, and delayed recall $(\mathrm{P}<0.28)$. The data from the prospective memory task (reminding task) $[\mathrm{F}(1,36)=8.71$, MSE $0.36, \mathrm{P}<0.006]$ showed that the chronic stress group (mean 2.42) was less likely than the controls (mean 1.84) to remember to remind the experimenter about their address at the end of the test session.

Semantic memory. A 2 (group: chronic stress, control) $\times 2$ (fluency measure: category, letter) ANOVA with a repeated measure for the last factor showed a main effect for group $[\mathrm{F}(1,36)=11.13$, MSE 171.45, $\mathrm{P}<0.002]$. The main effect of the fluency measures was also significant $[F(1,36)=30.18$, MSE 51.54, $\mathrm{P}<0.001]$ and therefore indicated that more words were generated in letter fluency (mean 50.65) than in category fluency (mean 41.61). More interestingly, the group $\times$ fluency measure interaction was significant $[\mathrm{F}(1,36)=11.35$, MSE 51.54, $\mathrm{P}<0.002]$, for which the follow-up t-tests revealed that the chronic stress patients did not differ from the controls with respect to category fluency $(\mathrm{P}>0.15)$, but were outperformed by the controls in letter fluency $(\mathrm{P}<0.001)$.

\section{Cognitive correlates of chronic stress}

The preceding analyses demonstrated suboptimal performance for $8\left(T_{2}\right.$ and $T_{3}$ from Buschke selective reminding, 2 measures of episodic memory under divided attention, the prospective memory task, letter fluency, digit symbol, and TMT B) out of 24 cognitive measures among the chronically stressed patients. In order to investigate which of these cognitive tests best

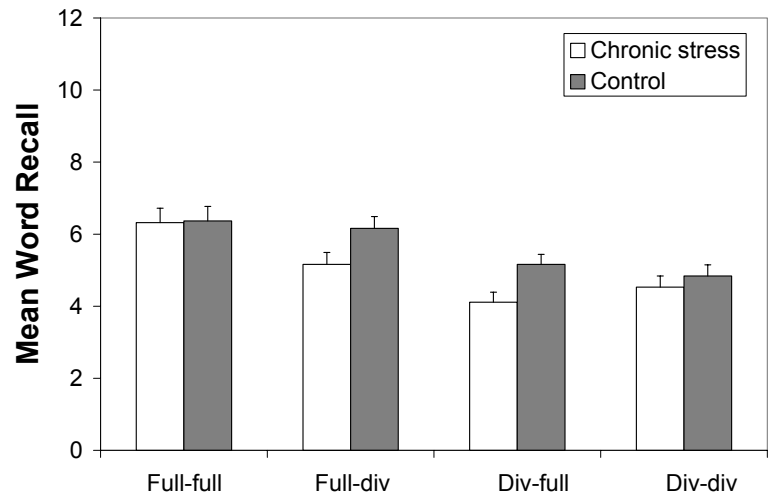

Figure 3. Means and standard errors of the means for recalled words as a function of group and the divided attention condition. (div = divided)

Table 3. Predictor variables for chronic stress in the logistic regression model.

\begin{tabular}{lcccc}
\hline Variable & $\beta$ & SE & Wald & $\operatorname{Exp}(\beta)$ \\
\hline Letter fluency & 1.27 & 0.49 & $6.85^{\mathrm{a}}$ & 3.57 \\
Reminding task (prospective memory) & 1.04 & 0.55 & $3.57^{\mathrm{b}}$ & 2.84 \\
\hline
\end{tabular}

a $P<0.01$.

${ }^{b} \mathrm{P}<0.05$, according to a forward stepwise (Wald) logistic regression.

differentiated the groups into chronically stressed patients and controls, a logistic regression was conducted with the diagnostic category as the outcome variable. We included six of the eight cognitive measures in the analysis. Two memory measures (Buschke $\mathrm{T}_{3}$, and the full-divided condition in the episodic memory task with divided attention) were excluded because of the high correlations with the two remaining measures in the analysis of episodic memory (Buschke $\mathrm{T}_{2}$, divided-full). These six measures were entered in a stepwise forward order fashion. All of the continuous variables were converted into $(Z)$ scores before the analysis in order to facilitate comparisons of the relative importance of the variables. The full model was statistically significant $\left(\chi^{2}=19.87, \mathrm{df}=2, \mathrm{P}<0.001\right)$. This model accounted for between $41 \%$ and $54 \%$ of the variance among the participants, with $84 \%$ of the patients successfully predicted to be chronically stressed and $79 \%$ of the controls correctly predicted to be controls. Overall, $82 \%$ of the predictions were accurate. As can be seen in table 3, letter fluency and the prospective memory task were the two tests that best distinguished between the controls and the chronically stressed participants.

\section{Discussion}

The following questions were addressed in this study. Do chronic stress patients report more subjective memory 
complaints than a control group? Do chronic stress patients show inferior cognitive performance in standard neuropsychological tests when compared with controls? If so, are deficits more salient under test conditions that are high in executive control? Finally, which cognitive measures are the most strongly related to chronic stress?

The results showed that the chronically stressed patients reported more subjective memory complaints than the controls; this finding agrees with previous findings from Sandström et al (12) and Van der Linden et al (18). With regard to the second question, the results demonstrated a pattern of suboptimal, as well as preserved, performance levels in standard neuropsychological tests among the chronic stress patients relative to the performance of the controls. This finding indicates that subjective reports of memory problems may reflect objectively measured cognitive deficits. Specifically, inferior performance levels were found for digit symbol, TMT B, letter fluency, and prospective memory. These measures have all been found to be related to executive control functioning and the prefrontal lobes $(37,46)$. Noteworthy is that we observed a substantial difference between the groups in letter fluency but not in category fluency. Although both category and letter fluency tasks are dependent upon executive control, recent studies have shown that category fluency relies more on the integrity of semantic memory (46-48). Thus the selective deficit for letter fluency and the relatively spared performance in the category fluency task in the chronic stress group are in line with less efficient executive control.

In addition to the standard neuropsychological approach, we also included two episodic memory tasks in which executive control was manipulated. In agreement with our expectation, the results from the selective reminding task showed a significant interaction between group and trial, indicative of less-pronounced learning across trials for the chronic stress patients than for the controls. Importantly, no differences in episodic memory performance were observed in the first learning trial, but, in the subsequent trial, the controls gained more than the chronic stress patients. Hence, the deficits were revealed only under task conditions assumed to require more strategic organization. This pattern of result may suggest inefficient strategy implementation for the chronic stress group that is reflective of suboptimal executive control processes. Previous research comparing populations with assumed prefrontal-lobe dysfunction have yielded similar results, namely, that learning across trials in episodic memory tasks has been compromised (49, 37).

Furthermore, the findings from the episodic memory and divided attention tasks yielded inferior performance in the chronic stress group under two of the three divided attention conditions. Specifically, no performance differences were found in the episodic memory task with no dual-task requirements, which mirror the results from trial 1 in the aforementioned episodic memory task. When attention was divided, either at encoding or retrieval, performance dropped disproportionally for the chronic stress group, whereas both the control group and the chronic stress group were equally penalized by divided attention delivered both at encoding and retrieval. Hence, memory deficits were the most evident for the chronic stress group when the executive control of attention was challenged by dual-task conditions. It is interesting to note that the chronic stress group was only disadvantaged under the task condition requiring divided attention at either encoding or retrieval and not under the task condition requiring divided attention during both encoding and retrieval. One obvious difference between these three conditions is that the encoding and retrieval requirements differ for the full-divided and divided-full conditions when compared with the divided-divided condition in that the former two conditions offer different processing contexts for encoding and retrieval by asking the participant to stop or begin sorting cards while encoding and retrieving words but, in the divided-divided condition, the task is the same at both encoding and retrieval. To be performing under the full-divided or divided-full condition, in which a processing shift is required, may perhaps add complexity to the task that may be more disruptive for a chronic stress group.

Moreover, no differences were found between the groups in the standard neuropsychological visuospatial episodic memory test, RCFT, for which encoding and retrieval of the Rey figure was relatively unconstrained, allowing the participants to devote full attention to the task. However, it should be noted that Sandström et al (12) found deficits in this task for a burnout group. In addition, the episodic memory task that best differentiated the groups was the prospective memory task, in which the participants had to remember to perform an action at an appropriate future occasion. Previous research has suggested that prospective memory relies on executive control functioning and serves as a good index of everyday memory functioning $(50,51)$. Overall the results from the episodic memory domain suggest that memory performance was suboptimal under conditions requiring allocation of strategic resources, and not under less-constrained task conditions.

Finally, no performance differences were found in basic measures of perceptual and motoric speed, reflective of intact basic processing operations. This pattern of spared and impaired cognitive functions highlights the fact that deficits accompanying work-related chronic stress may be selective in nature.

The results from the logistic regression identified two tasks, letter fluency and prospective memory, as 
being the most strongly associated with the stress diagnosis. Both of these tasks, as administered in this study, are easy to administer; thus they are good candidates for being included in a clinical screening battery for chronic stress.

Some limitations of our study need to be addressed. As has been suggested in previous research, reduced motivation or higher fatigability among these patients can be a plausible factor providing an alternative explanation for our findings, especially considering that low motivation and fatigability are integral parts of the chronic stress syndrome. Although these factors cannot be ruled out, we do find it less likely for two reasons. First, performance in the chronic stress group was not generally poorer than that of the controls; instead the deficits were selective to specific cognitive domains and task conditions. Second, performance did not decrease across the test session, as would have been predicted if fatigue built up over time during the testing.

Furthermore, we would like to draw attention to the fact that the use of SMBQ (29) for recruiting participants for our study may have had bias towards selecting people with more cognitive concerns, and, if so, may have increased the likelihood of detecting cognitive deficits in our sample. In regard to this issue, it is important to note that cognitive weariness is one of four subscales and that the stress group differed substantially on all four subscales of the SMBQ (29).

The cross-sectional nature of this small clinical study also limited our conclusions as to whether deficient executive functioning is a cause or a consequence of workrelated chronic stress. To help overcome some of these limitations, future studies should explore the cognitive consequences of work-related chronic stress in nonclinical population-based longitudinal samples.

In summary, our findings suggest cognitive deficits in chronically stressed patients that support dysexecutive control functioning, which in turn may implicate prefrontal system involvement. These results are in accordance with those recently shown for other stressrelated disorders, such as posttraumatic stress disorder (23), chronic fatigue syndrome (22), and the burnout syndrome $(12,18)$. An avenue for future research is to replicate and extend our findings by delineating the cognitive similarities and differences between stress-related conditions, as well as by improving our understanding of the underlying neuropathological mechanisms in chronic stress conditions.

To end, an understanding of the neuropsychological sequel of chronic stress may have implications for future treatment plans. As we know, executive processes are of particular importance with respect to rehabilitation because they are presumed to underlie many of the complex behaviors necessary for both functional independence and social integration.

\section{Acknowledgments}

We are thankful to Dr Marie Granlund and Professor Lars Nyberg for their valuable suggestions regarding the study.

The study was supported by grants from the Swedish Council for Working Life and Social Research, Dnr 2003-0761, awarded to Lisbeth Slunga Birgander and the Betula Study.

\section{References}

1. Schaufeli W, Enzmann D. The burnout companion to study \& practice, a critical analysis. Padstow (United Kingdom): International Ltd; 1998.

2. Melamed S, Ugarten U, Shirom A, Kahana L, Lerman Y, Froom P. Chronic burnout, somatic arousal and elevated cortisol levels. J Psychosom Res. 1999;46:591-8.

3. Spiegel K, Leproult R, Van Cauter E. Impact of sleep debt on metabolic and endocrine function. Lancet. 1999;354:143539.

4. McEwen BS, Sapolsky RM. Stress and cognitive function. Curr Opin Neurobiolog. 1995;5:205-16.

5. Meier ST. The construct validity of burnout. J Occup Psychol. 1984;57:211-9.

6. Shirom A, Ezrachi Y. On the discriminant validity of burnout, depression and anxiety: a re-examination of the burnout measure. Anxiety Stress Coping. 2003;16:83-97.

7. Prins LB, van der Meer JWM, Bleijenberg G. Chronic fatigue syndrome. Lancet. 2006;367:346-55.

8. Quinter JL, Milton LC. Fibromyalgia falls foul of a fallacy. Lancet. 1999;353:1092-94

9. Ursin H, Eriksen HR. Sensitization, subjective health complaints, and sustained arousal. Ann NY Acad Sci. 2001;933:119-29.

10. Wessely S, Nimnuan C, Sharpe M. Functional somatic syndromes: one or many? Lancet. 1999;354:936-9.

11. Maslach C, Schaufeli WB, Leiter MP. Job burnout. Annu Rev Psychol. 2001:52;397-422.

12. Sandström A, Nyström Rhodin I, Lundberg M, Olsson T, Nyberg L. Impaired cognitive performance in patients with chronic burnout syndrome. Biol Psychol. 2005;69:271-9.

13. McEwen BS. Protective and damaging effects of stress mediators. N Eng J Med. 1998;338:171-9.

14. Lupien SJ, Lepage M. Stress, memory, and the hippocampus: can't live with it, can't live without it. Behav Brain Res. 2001;127:137-58

15. Radley JJ, Sisti HM, Hao J, Rocher AB, McCall T, Hof PR, et al. Chronic behavioral stress induces apical dendritic reorganization in pyramidal neurons of the medial prefrontal cortex. Neurosci. 2004;125:1-6.

16. Alderson AL, Novack TA. Neuropsychological and clinical aspects of glucocorticoids and memory: a review. J Clin Exp Neuropsychol. 2002;24:335-55

17. Pruessner JC, Hellhammer DH, Kirschbaum C. Burnout, perceived stress, and cortisol responses to awakening. Psychosom Med. 1999;61:197-204.

18. Van der Linden D, Keijsers GPJ, Eling P, van Schaijk R. Work stress and attentional difficulties: an initial study on burnout and cognitive failures. Work Stress. 2005;19:23-36. 
19. Braver TS, Barch DM. A theory of cognitive control, aging cognition and neuromodulation. Neurosci Biobehav Rev. 2000;26:809-17.

20. Miyake A, Friedman NP, Emerson MJ, Witzki AH, Howerter A. The unity and diversity of executive functions and their contributions to complex frontal lobe tasks: a latent variable analysis. Cognit Psychol. 2000;41:49-100.

21. Miller EK, Cohen JD. An integrative theory of prefrontal cortex function. Annu Rev Neurosci. 2001;24:167-202.

22. Capurun L, Welberg L, Heim C, Wagner D, Solomon L, Papanicolaou DA, et al. Cognitive dysfunction relates to subjective report of mental fatigue in patients with chronic fatigue syndrome. Neuropsychopharmacology. 2006;31:1777-84

23. Koenen KC, Driver KL, Oscar-Berman M, Wolfe J, Folsom S, Huang MT, et al. Measures of prefrontal system dysfunction in posttraumatic stress disorder. Brain Cogn. 2001;45:64-78.

24. Binder LM, Rohling ML, Larrabee GJ. A review of mild head trauma, part 1: meta-analytic review of neuropsychological studies. J Clin Exp Neuropsychol. 1997;19:421-31.

25. Nyberg L, Nilsson L-G, Olofsson U, Bäckman L. Effects of division of attention during encoding and retrieval on age differences in episodic memory. Exp Aging Res. 1997;23:137-43.

26. Mangels JA, Craik FIM, Levine B, Schwartz ML, Stuss DT. Effects of divided attention on episodic memory in chronic traumatic brain injury: a function of severity and strategy. Neuropsychologia. 2002;40:2369-85.

27. Bergdahl J, Larsson A, Nilsson L-G, Riklund Åhlström K, Nyberg L. Treatment of chronic stress in employees: subjective, cognitive, and neural correlates. Scand J Psychol. 2005;46:395-402.

28. Moscovitch M, Winocur G. The frontal cortex and working with memory. In: Stuss DT, Knight RT, editors. Principals of frontal lobe function. New York (NY): Oxford University Press; 2002. p 188-209.

29. Melamed S, Kushnir T, Shirom A. Burnout and risk factors for cardiovascular diseases. Behav Med. 1992;18:53-60.

30. Grossi G, Perski A, Evengård B, Blomkvist V, Orth-Gomér K. Physiological correlates of burnout among women. J Psychosom Res. 2003;55:309-16.

31. Melamed S, Shirom A, Toker S, Berliner S, Shapira, I. Burnout and risk of cardiovascular disease: evidence, possible causal paths and promising research directions. Psychol Bull. 2006;132:327-53.

32. Dureman I, Kebbon L, Österberg E. Manual till DS-batteriet [manual to the DS-battery]. Stockholm: Skandinaviska Testförlaget; 1971

33. Nilsson L-G, Bäckman L, Erngrund K, Nyberg L, Adolfsson R, Bucht G, et al. The Betula prospective cohort study: memory, health, and aging. Aging Neuropsychol Cogn. 1997;4:1-32.

34. Mäntylä T. Assessing absentmindedness: prospective memory complaint and impairment in middle-aged adults. Mem Cog. 2003;31:15-25.

35. Wechsler D. Wechsler Memory Scale—revised manual. San Antonio (TX): The Psychological Corporation; 1987.

36. Reitan RM. Trail making test. Tucson (AZ): Reitan Neuropsychology Laboratory; 1992

37. Lezak MD. Neuropsychological assessment. Oxford (United Kingdom): University Press Inc; 1995.

38. Salthouse TA, Babcock RL. Decomposing adult age differences in working memory. Dev Psychol. 1991;27:763-76.

39. Salthouse TA. Speed mediation of adult age differences in cognition. Dev Psychol. 1993;29:722-38.

40. Buschke H. Selective reminding for analysis of memory and learning. J Verbal Learn Verbal Behav. 1973;12:543-50.

41. Murdock BB Jr. Effects on a subsidiary task on short-term memory. Br J Psychol. 1965;56:413-9.

42. Rey A. L'examen psychologique dans les cas d'encephalopathie traumatique. Arch Psychol 1941;28:286-340; (For translation, see J Corwin, \& FW Bylsma. Clin Neuropsychol. 1993;7:4 9.)

43. Meyers JE, Meyers KR. Rey Complex Figure Test and Recognition Trial: professional manual. Odessa (FL): Psychological Assessment Resources Inc; 1995.

44. Mäntylä T, Nilsson L-G. Remembering to remember in adulthood: a population-based study on aging and prospective memory. Neuropsychol Cogn. 1997;4:81-92.

45. Benton AL, Hamsher K deS. Multilingual aphasia examination. Iowa City (IA): AJA Associates; 1989.

46. Henry JD, Crawford JR, Phillips LH. Verbal fluency performance in dementia of the Alzheimer's type: a meta-analysis. Neuropsychologia. 2004;42:1212-22.

47. Paulesu E, Goldacre B, Scifo P, Cappa SF, Gilardi MC, Castiglioni I, et al. Functional heterogeneity of left inferior frontal cortex as revealed by fMRI. NeuroReport. 1997;8:2011-6.

48. Mungas D, Jagust WJ, Reed BR, Kramer JH, Weiner MW, Schuff N, et al. MRI predictors of cognition in subcortical ischemic vascular disease and Alzheimer's disease. Neurology. 2001;57:2229-35.

49. Nyberg L, Sandblom J, Jones S, Stigsdotter Neely A, Petersson KM, Ingvar M, et al. Neural correlates of training-related memory improvement in adulthood and aging. Proc Natl Acad Sci U S A. 2004;100:13728-33.

50. McDaniel MA, Glisky EL, Rubin SR, Guynn MJ, Routhieaux BC. Prospective memory: a neuropsychological study. Neuropsychology. 1999;13:103-10.

51. Kliegel M, Eschen A, Thöne-Otto AIT. Planning and realization of complex intentions in traumatic brain injury and normal aging. Brain Cogn. 2004;56:43-54.

Received for publication: 21 January 2006 